\title{
Recognition of Handwritten Characters using Deep Convolutional Neural Network
}

\author{
Jagan Mohan Reddy D, A Vishnuvardhan Reddy
}

\begin{abstract}
Handwritten character recognition (HCR) mainly entails optical character recognition. However, HCR involves in formatting and segmentation of the input. HCR is still an active area of research due to the fact that numerous verification in writing style, shape, size to individuals. The main difficult part of Indian handwritten recognition has overlapping between characters. These overlapping shaped characters are difficult to recognize that may lead to low recognition rate. These factors also increase the complexity of handwritten character recognition. This paper proposes a new approach to identify handwritten characters for Telugu language using Deep Learning $(D L)$. The proposed work can be enhance the recognition rate of individual characters. The proposed approach recognizes with overall accuracy is $94 \%$.
\end{abstract}

\section{INTRODUCTION}

Handwritten recognition can be done in two ways viz online and offline. The offline is recognition system performed after the digits are digitized. On the other hand, online recognition system can maintain real time information such as stroke. It is generally written on a pressure sensitive surface from which the stroke is preserved [1]. Off-line handwriting recognition is the process to translate digital image of handwritten text into machine editable text. Several recognition methodologies of recognition of English, Latin, Arabic, Chinese scripts are extensively reviewed in [1], [2], [3], [4].

Telugu is the Dravidian language and is third most popular script in India [5]. It is also an official language of the southern Indian popularly used in state, Andhra Pradesh and its neigh-boring states. It is similar to the Kannada language. Telugu is a syllabic language and represents a complete syllable. Forally, there are 10 numerals, 18 vowels, 36 consonants, and three dual symbols. The challenging part of Indian handwritten character recognition is shape similarities among different characters. Handwritten recognition is very complex as there is a tiny variation in writing, which leads to great complex in recognition. The handwritten style of an individual different in size and shape which leads various numerals leads to similar shape. India is a multilingual and multi-script coun-try comprising of twenty five official languages [6], namely Assamese, Bangla, Bodo, Dogri, English, Gujarati, Hindi, Konkani, Kannada, Kashmiri, Malayalam, Maithili, Manipuri, Marathi, Nepali, Oriya, Punjabi, Rajasthani, Santali, Sanskrit, Sindhi, Tamil, Telugu and Urdu. Recognition of handwrittenIndian scripts is more complicated than Roman

Revised Manuscript Received on April 12, 2019.

Jagan Mohan Reddy D,Department of Computer Science \& Engineering, Lakireddy Bali Reddy College of Engineering, Mylavaram, Andhra Pradesh, India - 521230 (Email: jagan.reddy507@gmail.com)

Dr. M Siva Koti Reddy,Department of Computer Science \& Engineering G Pulla Reddy College of Engineering Kurnool, Andhra Pradesh, India - 518007 (Email: avijavishnu@gmail.com) script because of the presence of compound characters and vowel modifiers.

To recognize optical characters, features are extracted from every numeral that is captured. Each numeral has it own pattern in certain class. As may advancement in the optical character recognition, still the demand is high among research community. Earlier methods for recognition of handwritten character includes directional distance features [7], gradient-based features [8], wavelet-based features [9], pixel distance features [10], convex hull based features [11]. In recent times, with the help Deep Learning (DL) the the recognition of objects has more advancements. However, to discriminate the the features is not understood well for various languages. This need to investigate and identify newer patterns with less complexity algorithms that can classify accurately for complex style of handwritten characters.

The objective of this work is to effectively extract the topo-logical features for handwritten numerals recognition using convolutional Neural Network (CNN) with deep network. The rest of the paper is organized as follows. Section II describes the present work and its methodology. The experimental setup is discussed in III with discussions. In Section IV concludes with remarks of proposed work in the end.

\section{PROPOSED FRAMEWORK TO RECOGNIZEHANDWRITTEN NUMERALS FOR TELUGU}

In this section, we discuss the proposed method to recognize handwritten numerals. The framework involves several stages to accurately identify digits. Fig.1 describes the proposed schematic overview of our framework. It has several phases:

Step i collected Telugu Handwritten Samples from 280 in-dividuals which are in different styles and shapes.

Step ii Collected samples are pre-processed to scale the images by $32 \times 32$ in gray scale format.

Step iii The dataset is divided into two groups as train and test phase. In training phase, we have considered 200 individual samples i.e 2000 numerals and for testing phase 80 individual samples i.e. 800 numerals.

Step iv We implemented a Convolutional Neural Network (CNN) model in Python. The training samples are input to $\mathrm{CNN}$ and that adjusted the weights to accu-rately recognize digits.

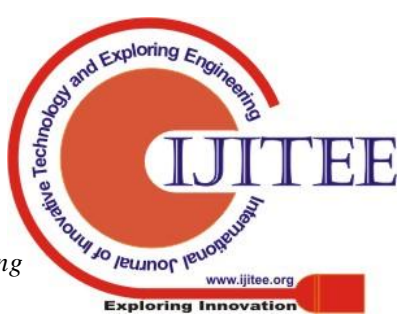




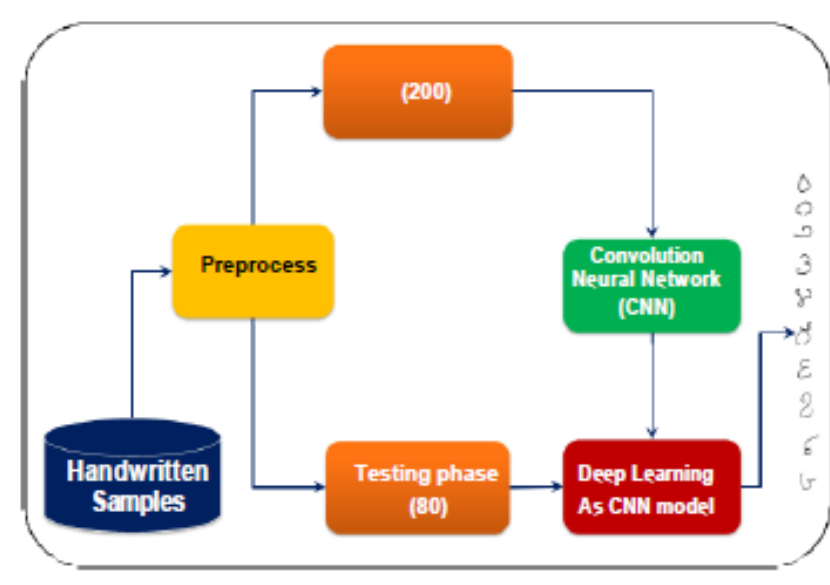

The deep network is created based on the [12]. Our work utilized the LeNet-5 architecture to recognize the handwritten digits. It has several stages viz. convolution, sub-sampling and fully connected layer. In this architecture, the image is scaled to $32 \times 32$. The scaled image forwarded to convolution layer, where there are 6 kernel functions are applied with a size of $5 \times 5$. These kernels are extracted from the image itself which can detect various edges from image. The mathematical notation of convolutional operation is given in Equ. 1.

The next layer, is a sub-sampling that reduce the dimensional of the image with $2 \times 2$ map pooling and a stride of 2 with of every kernel function. The sub-sampling is denoted mathematically in Equ,2. This will be repeated until the input size is $5 \times 5$ with 16 kernel functions that creates a fully connected network of 120 nodes and further reduced to 84 features of every digit. The architecture is shown in Fig. 2.

Where " $n$ " is the dimensions of input image, $p$ is the padding and $s$ is the stride.

Where " $\mathrm{n} \mathrm{h}$ and $\mathrm{N} w$ " is the height and width of input image, $\mathrm{s}$ is the stride and " $\mathrm{c}$ is the number of channels".

The proposed method CNN model is implemented in Python that can accurately recognize the digits. This procedure re-quires both training and testing patterns involves transformed into gray scale and image re-size an normalized each image. Then the training function will call to build a CNN model and supplies the test samples to measure the performance of recognition rate. In $\mathrm{CNN}$, the loss function and optimization of weights are calculated at every epoch with a certain direction is described in Algorithm 1.

Telugu language is one of the popular in south India. The typical handwritten samples are shown in Fig. 3. These samples are pre-processed and segmented into gray scale images.

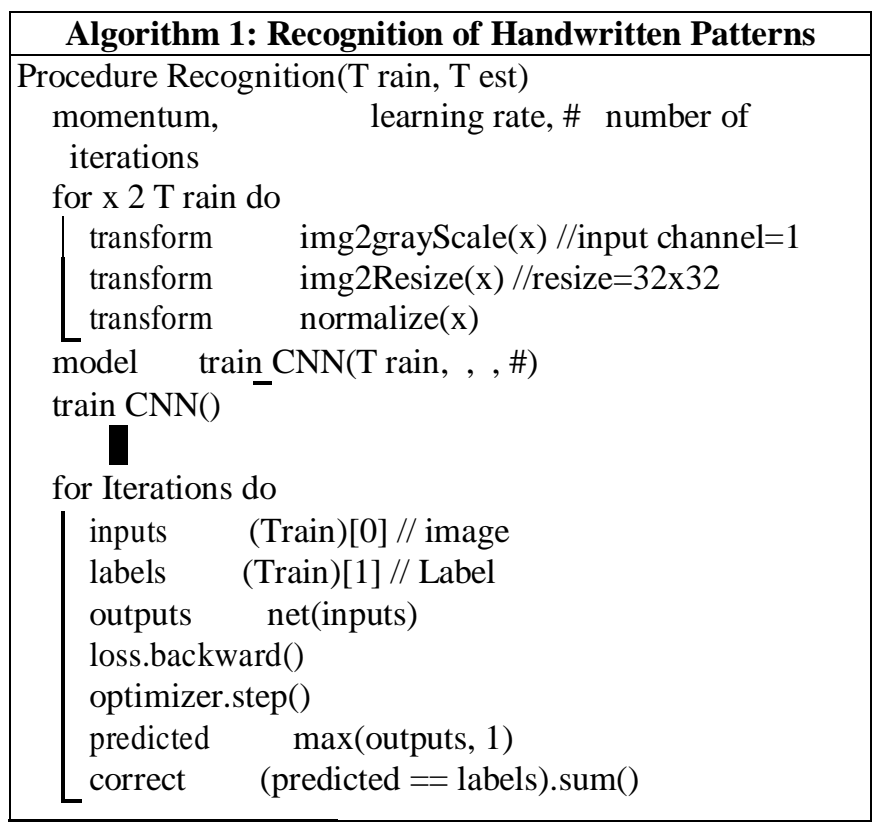

A. Description of Telugu Handwritten Numerals dataset The dataset collection process carefully designed and main-tained diversity of across the gender. It has collected man-ually from 280 individuals with various handwritten styles. The collected samples are digitized and segmented to create numeral database. While segmentation the image are scaled to $32 \times 32$ with out any loss of information. With the diversity of writing style, handwritten recognition allows us to aimed to process and extract different features that can distinguish between numerals. Part of the dataset obtained from [13].

\section{EXPERIMENTAL RESULTS AND DISCUSSIONS}

In this section, we describe the experimental setup to examine the performance of proposed method in recognition of handwritten numerals of Telugu and also measures the clas-sification rate of each numeral with $\mathrm{CNN}$ based classifiers. The design of a classifier is involved several steps that can create a deep network to recognize the numerals. The network has convolutional operation and sampling operations are involved. Given any 32x32 image will be reduced to 84 features that can be distinguish each digit. The deep network extracts the features based on the weights of each kernel derived from within a portion of image.

The proposed method then evaluated on test dataset to measure the overall accuracy and individual digit recognition rate. We ran different iterations by varying the neural network parameters that can observed in lot of variation in accuracy. There are total of 2000 samples are used to build a model and 800 samples are used to recognize the new handwritten samples. 
The overall accuracy is captured of different epochs is shown in Fig. 4. At the $50^{\text {th }}$ epoch, we measured heights accuracy of $94 \%$ is captured. In our experiments, we also

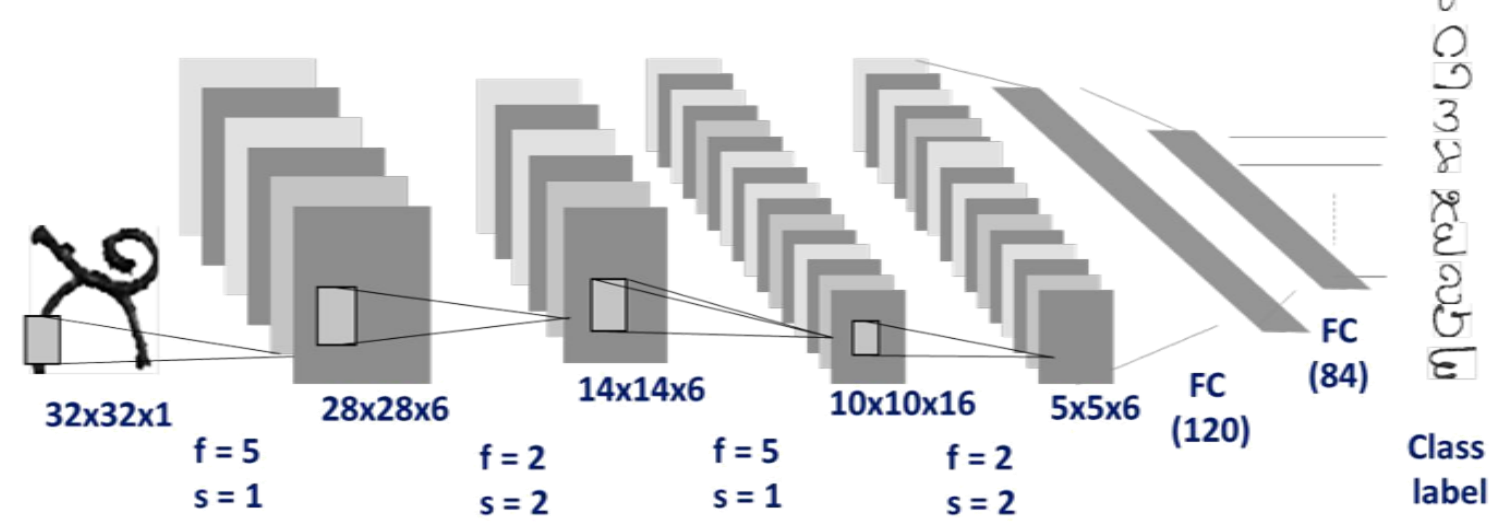

Fig. 2. Deep network for Telugu numerals [12]

\begin{tabular}{|c|c|c|c|c|c|c|c|c|c|}
\hline 0 & 1 & 2 & 3 & 4 & 5 & 6 & 7 & 8 & 9 \\
\hline 0 & 0 & 0 & 3 & $\gamma$ & $\phi$ & $\varepsilon$ & 8 & $G$ & $\varepsilon$ \\
\hline 0 & 0 & J & 3 & 8 & 8 & $\varepsilon$ & 2 & $G$ & $\varepsilon$ \\
\hline 0 & 0 & ט & 3 & 8 & 8 & $\varepsilon$ & 8 & $G$ & $\varepsilon$ \\
\hline 0 & 0 & $v$ & 3 & 8 & $\pi^{\circ}$ & $\varepsilon$ & 2 & $G$ & $\varepsilon$ \\
\hline 0 & $\Omega$ & 2 & 3 & 8 & $\pi$ & $\varepsilon$ & 8 & $v$ & $\varepsilon$ \\
\hline$a$ & 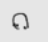 & అ & 3 & 8 & $x$ & $\varepsilon$ & 2 & $v$ & $\varepsilon$ \\
\hline 0 & 0 & ـ & 3 & $\gamma$ & $x$ & $\varepsilon$ & 2 & $G$ & $\varepsilon$ \\
\hline 0 & 0 & 0 & 3 & $\gamma$ & 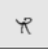 & $\varepsilon$ & 2 & $G$ & $\varepsilon$ \\
\hline 0 & 0 & ט & 3 & $\gamma$ & $\pi$ & $\varepsilon$ & 2 & G & $\varepsilon$ \\
\hline 0 & $\cap$ & 0 & 3 & 8 & $x$ & $\varepsilon$ & 2 & $G$ & $\varepsilon$ \\
\hline
\end{tabular}

Fig. 3. Diversity in handwritten numerals of Telugu language

captured the each digit recognition rate that gives the insight of how each digits can be accurately identified. Most of the digits are recognized more the $95 \%$ except digit 4 , digit 7 and digit 9 are very low in accuracy due to ambiguity in patterns. These digits are closely resemble each other that can successfully recognize individual patterns. The highest recognition rate is captured digit is " 3 " 3 with $98 \%$. The detailed accuracy of each digit is shown in Fig. 5.

\section{CONCLUSION}

An efficient handwritten character recognition approach is proposed in this work. The proposed work employed a deep network model to recognize the handwritten numerals. The experimental results shown that convolutional neural networks performance better in recognition for Telugu language. We have captured the low recognition rate of three digits viz. 3,7 and 9 can be improved by introducing the new kernel methods. The possible reason may be a overlapping patterns among them. However, the recognition rate can be increase by introducing quad-tree based structure that can even deep understanding of each digit patterns at coarser-grain level. Further, a newer methods an be used to explore topological features to improve the accuracy. This work will be extend to recognize Telugu characters too.

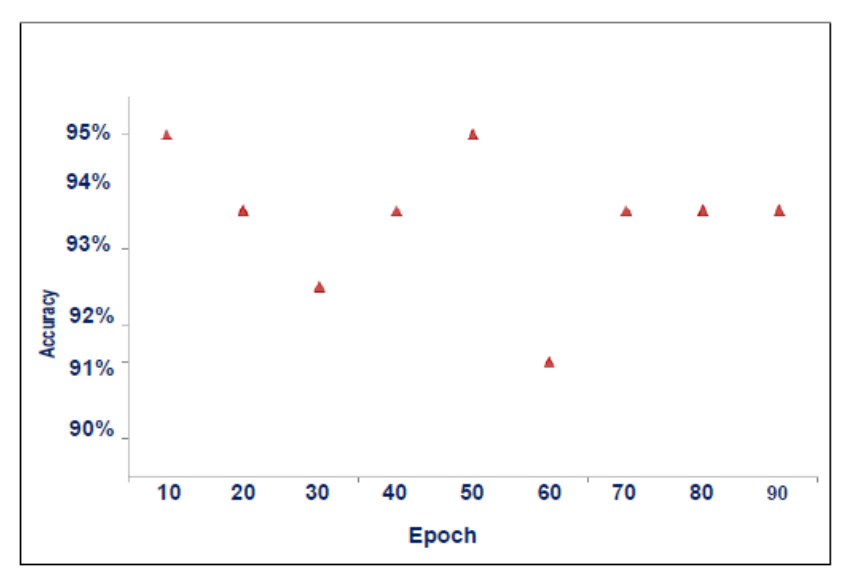

Fig. 4. Recognition rate

\section{ACKNOWLEDGMENT}

Authors are thankful to the LBRCE, "Research Center" recognized by the JNTU, Kakinada for providing infrastructure facilities during the progress of work.

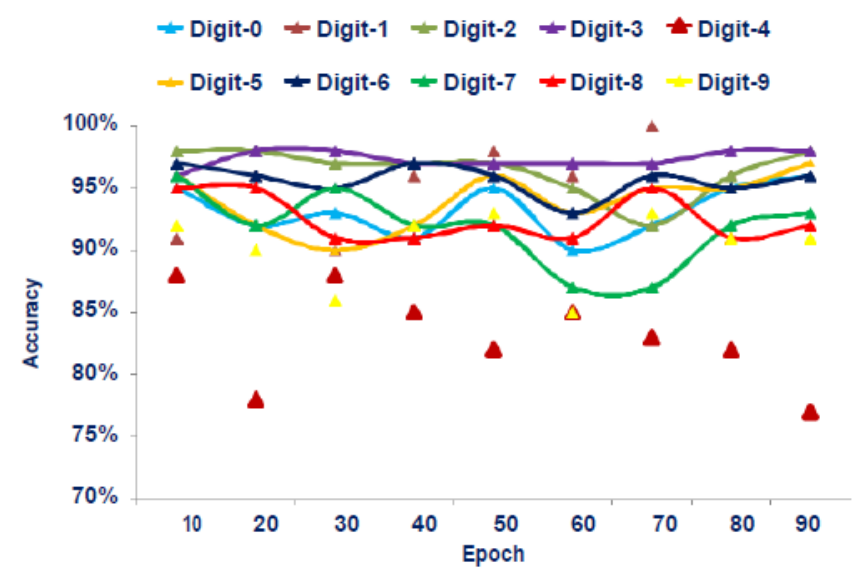

Fig. 5. Recognition rate of individual numeral 


\section{REFERENCES}

1. R. Plamondon and S. N. Srihari, "Online and off-line handwriting recognition: a comprehensive survey," IEEE Transactions on pattern analysis and machine intelligence, vol. 22, no. 1, pp. 63-84, 2000.

2. N. Arica and F. T. Yarman-Vural, "An overview of character recognition focused on off-line handwriting," IEEE Transactions on Systems, Man, and Cybernetics, Part C (Applications and Reviews), vol. 31, no. 2, pp. 216-233, 2001.

3. L. M. Lorigo and V. Govindaraju, "Offline arabic handwriting recog-nition: a survey," IEEE transactions on pattern analysis and machine intelligence, vol. 28, no. 5, pp. 712-724, 2006.

4. G. Nagy, "Chinese character recognition: a twenty-five-year retrospec-tive," in Pattern Recognition, 1988., 9th International Conference on. IEEE, 1988, pp. 163-167.

5. S. Rajashekararadhya and P. V. Ranjan, "Neural network based hand-written numeral recognition of kannada and telugu scripts," in TENCON 2008-2008 IEEE Region 10 Conference. IEEE, 2008, pp. 1-5.

6. —_ "Efficient zone based feature extration algorithm for handwritten numeral recognition of four popular south indian scripts." Journal of Theoretical \& Applied Information Technology, vol. 4, no. 12, 2008.

7. H. Fujisawa and C.-L. Liu, "Directional pattern matching for character recognition revisited," in null. IEEE, 2003, p. 794.

8. C.-L. Liu, K. Nakashima, H. Sako, and H. Fujisawa, "Handwritten digit recognition: benchmarking of state-of-theart techniques," Pattern recognition, vol. 36, no. 10, pp. 2271 $2285,2003$.

9. S. E. Correia, J. M. de Carvalho, and R. Sabourin, "On the performance of wavelets for handwritten numerals recognition," in Pattern Recog-nition, 2002. Proceedings. 16th International Conference on, vol. 3. IEEE, 2002, pp. 127-130.

10. I.-S. Oh and C. Y. Suen, "A feature for character recognition based on directional distance distributions," in Document Analysis and Recog-nition, 1997., Proceedings of the Fourth International Conference on, vol. 1. IEEE, 1997, pp. 288-292.

11. N. Das, S. Pramanik, S. Basu, P. K. Saha, R. Sarkar, M. Kundu, and M. Nasipuri, "Recognition of handwritten bangla basic charac-ters and digits using convex hull based feature set," arXiv preprint arXiv:1410.0478, 2014.

12. Y. LeCun et al., "Lenet-5, convolutional neural networks," URL: http://yann. lecun. com/exdb/lenet, p. 20, 2015.

13. N. Das, J. M. Reddy, R. Sarkar, S. Basu, M. Kundu, M. Nasipuri, and D. K. Basu, "A statistical-topological feature combination for recognition of handwritten numerals," Applied Soft Computing, vol. 12, no. 8, pp. 2486-2495, 2012. 